\title{
Network Topology in Immune System Shape Space
}

\author{
John Burns and Heather J. Ruskin \\ School of Computing, \\ Dublin City University, \\ Dublin 9, Ireland \\ \{jburns, hruskin\}@computing.dcu.ie \\ http://www.dcu.ie/computing/msc/index.html
}

\begin{abstract}
We consider the emergent network topology of an immune system shape space at the end of primary response. We extend the formalism of shape space in order to model the relationship between activated immune lymphocytes and stimulant antigen presentation cells by way of a graph consisting of a pair $G=(V, E)$ of sets. The vertex set $V$ is the set of activated genotypes, while the edge set $E$ connects such activated immune lymphocytes and stimulant antigen presentation cell in shape space. This paper shows how shape space graph edge weighting can be viewed, from the biological perspective, as the vigour with which an activated cytotoxic immune cell suppresses the infected antigen presentation cell which stimulated it. In this research, we also identify critical vertices (called $\alpha$-vertices). These $\alpha$-vertices act as bridging vertices in that they join subgraphs of unrelated immune response. As a consequence of this, such $\alpha$-vertices ideally model immune cytotoxic lymphocyte memory cells. By representing memory cells as highly connected vertices, we show how such cells play a significant role in the elimination of pathogenic agents.
\end{abstract}

\section{Introduction}

In this paper we present results from recent work carried out to model the emergence of shape diversity within the immune system. Previously [1], we introduced a new process by which two formalisms, usually separately addressed, may be integrated. These formalisms are known as shape space and physical space. We highlighted a means by which localised dynamics effect global (or shape space) condition, and how global condition in turn may feed information down to local physical space. This approach is now further refined by treating shape space as a self-organising, dynamic network in 2-dimensional space. The system is considered to be exposed to a set of genetically varied pathogens in order to simulate normal human immune experience over a fixed period of time. We then study the cytotoxic lymphocyte activation patterns which emerge naturally in shape space. The results presented here show that, at the end of primary response, a network of activated cytotoxic lymphocytes and pathogen challengers emerges in shape space. 
The main contribution of this work is as follows: We present a means to model the genotype (or shape) space of the immune system as set of connected, directed and weighted subgraphs. These subgraphs, will, by way of the emergence of critical (or $\alpha$-) vertices, merge over time. We show that disruption to $\alpha$-vertex formation degrades immune response more severely than does the disruption of other (which we call $\beta$-) vertices. Disruption is likely to occur whenever a viral mutation is a factor, for example, Human Immune Virus (HIV) or Influenza. The means by which such graphs grow, and how rewiring of vertices improves response over time, is also investigated. This work demonstrates that edge weighting can be viewed, from the biological perspective, as the vigour with which an activated cytotoxic immune cell suppresses the infected antigen presentation cell which stimulated it. In shape space, this weighting is the distance (d) from $\alpha$ - and $\beta$ - vertices to the stimulant pathogen (effectively, it is the length of the edge).

\section{The Model}

In this section we first review some important features of our previous work, and introduce new detail. For a full exposition of both shape and physical space, the reader is directed to [4]. The shape space formalism was introduced by [5] as a way to represent antibody-antigen binding dynamics. Further research refined this model, notably [6] and 7]. The features of cytotoxic lymphocyte (CTL) cells and antigen presentation (APC) cells which govern the dynamics of cell binding (known as the antigenic determinant), may be represented by $N$ parameters. If the $N$ parameters are combined into a vector, the antigenic determinant for each APC and each CTL can be considered as points within an $N$-dimensional Euclidean space of length $L_{s s}$. Some notational conventions are observed in the work which follows (where upper-case letters refer to shape space, and lower case, to physical space), and this convention is summarised as follows:

1. $\left(C T L^{+}, c t l^{+}\right)$: activated cytotoxic lymphocyte cells which are ready to attack and remove infected antigen presentation cells. These cells are often referred to as armed effectors. The recirculation patterns of $\mathrm{ctl}^{+}$are different from $c t l^{-}$, in that $c t l^{+}$will leave the lymphatic compartment and migrate to the location of infection detection. Alteration of recirculation patterns is a common feature of cellular immune response in healthy [8] and diseased [9] immune systems.

2. $\left(A P C^{+}, a p c^{+}\right)$: infected antigen presentation cells (typically, dendritic cells) which, having engulfed a virus particle, has gone on to present characteristic viral peptide fragments on its surface.

At the start of each simulation, shape space is characterised by two (non-zero) subpopulations: $C T L^{-}$and $A P C^{+}$, representing the number of precursor cytotoxic lymphocyte and active infected antigen presentation cell genotypes respectively. A further subpopulation (called $C T L^{+}$), arises once an $A P C^{+}$is detected by a $C T L^{-} . C T L^{+}$are activated cytotoxic lymphocyte genotypes. The $C T L^{+}$ 
subpopulation level increases each time another detection occurs, such that the total $C T L^{-}$approaches 0 . Denoting CTL and APC genotype vectors as c and a respectively, we further develop shape space as follows: Surrounding each c is a disc of radius $\rho$ 1. Any a located within this disc will be subject to a clearance pressure inversely proportional to the distance $(d)$ between the $\mathbf{c}$ and $\mathbf{a}$ in shape space $(d=\|\mathbf{c}-\mathbf{a}\|)$. Our approach is to place into shape space an increasingly diverse set of antigen challenges and test the varying immune response.

Shape space may be further explored using graph theory [10] to model the relationship between $A P C^{+}$and $C T L^{+}$. In this approach, shape space is a graph consisting of a pair $G=(V, E)$ of sets satisfying $E \subseteq[V]^{2}$. The set of vertices $V$ is made up of both $C T L^{+}$and $A P C^{+}$. Edges connect an $A P C^{+}$to the set of $C T L^{+}$stimulated by its presence to become activated. Each $G$ is directed and weighted. An initial set of vertices is introduced at time $\tau_{0}$ based on model startup parameters. At any time $\tau_{k}>\tau_{0}$, a new vertex may be added in shape space with probability dependent both on an $a p c^{+}$and $c t l^{-}$being neighbours in physical space $(P(N))$ and the distance $(d)$ between $A P C^{+}$and $C T L^{-}$in shape space is less than or equal to some threshold $\hat{\rho}(P(d \leq \hat{\rho}))$. The outcome of both events are independent of each other, so the probability of a new vertex being added is: $P($ newvertex $)=P(N) P(d \leq \hat{\rho})$ for any given $\left\{A P C^{+}, C T L^{-}\right\}$conjugate. A newly added vertex is designated $C T L^{+}$(indicating it has been recruited from the $C T L^{-}$pool). Whenever a new vertex is added, an edge is added by joining the new vertex to the vertex which stimulated its activation. Thus the relationship represented by an edge between two vertices can be understood as: the $C T L^{+}$was recruited from the $C T L^{-}$pool by the presence of the $A P C^{+}$, and, the $C T L^{+}$acts against the stimulant $A P C^{+}$:

$$
C T L^{-} \stackrel{A P C^{+}}{\longrightarrow} C T L^{+}
$$

and

$$
C T L^{+ \text {attacks }} A P C^{+}
$$

respectively.

A new edge is added whenever a new vertex is, but also, whenever a new $\alpha$-vertex appears. An $\alpha$-vertex is one which although emerges in response to one individual $A P C^{+}$actually effects pressure on other $A P C^{+}$. As shown in Fig. 1 the $\alpha$-vertex acts against three $A P C^{+}$genotypes (as the $A P C^{+}$is the median vertex in each subgraph, we use the notation $m_{i}$ ). In so doing, it connects the otherwise unconnected subgraphs of $Q, R$ and $S$. The importance of the $\alpha$-vertex is clear: $\alpha$-vertices are promiscuous, not only targeting the stimulant infected cell but also other $A P C^{+}$nearby in shape space (Fig. 1, the $m_{1}, m_{2}$ and $m_{3}$ vertices, respectively). The emergence of such vertices marks a diversity threshold of immune response $\left(\alpha_{\text {crit }}\right)$ which once reached, favours a full and healthy clearance of infected cells from the lymphatic compartment. Such $\alpha$-vertices are unique in that they participate in Eqn.(2) without having first participated in Eqn.(11). Clearance pressure acts along directed edges which are defined by the triple:

\footnotetext{
${ }^{1}$ Clearly, with $N=2$, the area of this disc is $\pi \rho^{2}$.
} 


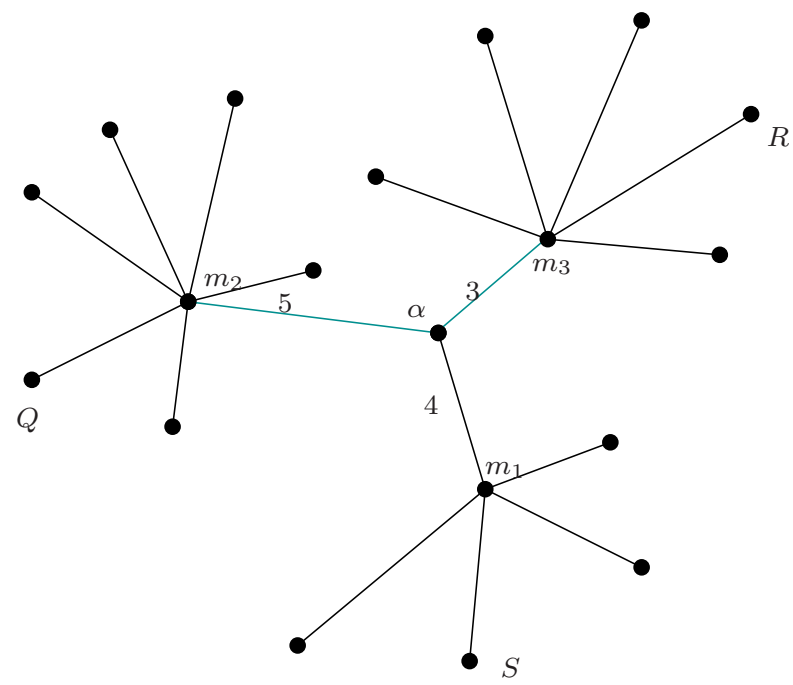

Fig. 1. Three subgraphs with median vertices (stimulant $A P C^{+}$) at $m_{i}$. Leaves $(\beta$ vertices) represent activated cytotoxic lymphocyte genotypes $\left(C T L^{+}\right)$. The $\alpha$-vertex is also a $C T L^{+}$, but is one which though originally activated by $m_{1}$ now affects clearance pressure against both $m_{2}$ and $m_{3}$.

$$
\vec{E}:=\{(e, x, y) \mid e \in E ; x, y \in V ; e=x y\}
$$

With respect to Fig. 1, the $\alpha$-vertex clearance pressure acts in three directions (for $i=1, . ., 3): \vec{e}_{i}=\left(e_{i}, \alpha, m_{i}\right)$.

Rewiring of edges happens whenever a subpopulation of $A P C^{+}$disappears, to be replaced by a later $A P C^{+}$. From the biological point of view, the disappearance of a subpopulation of $A P C^{+}$happens whenever the viral genotype challenge is completely eradicated. Rewiring can be viewed as a secondary immune response to some further antigenic challenge. Consistent with [11], we refer the $A P C^{+}$as the median vertex $\left(m_{i}\right)$ in a subgraph $S$. Rewiring is a three step process as follows:

(i) The median vertex $m_{i}$ is deleted from the graph and the vertices connected to it become disconnected.

(ii) A new median vertex $m_{j}(i \neq j)$ is introduced, representing a new viral infection in the system.

(iii) Each leaf 2 (or $\beta$-vertex) rewires to the new median $m_{j}$. Depending on the location of $m_{j}$ in shape space, there is a probability that not all leaves will reconnect to the median vertex (due to $d>\hat{\rho}$ ) and such leaves will remain unconnected until a more central vertex appears. In biological terms, the disappearance of a stimulus $A P C^{+}$usually results in a gradual decline of the effector response, to some small, non-zero level which remains as a form of immune memory.

\footnotetext{
${ }^{2}$ A leaf is a vertex of $\operatorname{deg}(1)$.
} 
(a)

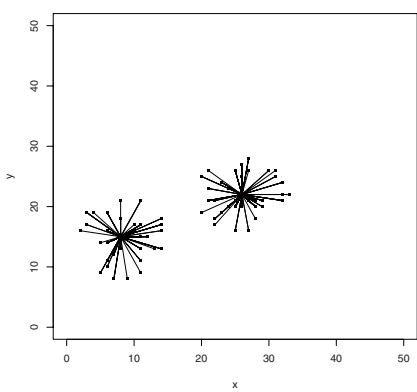

(c)

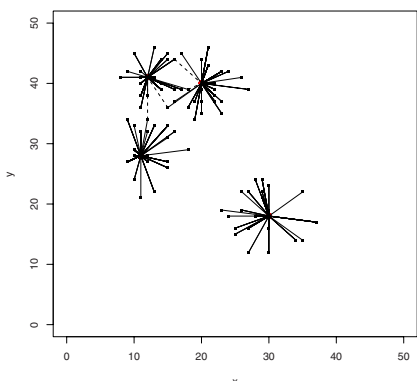

(b)

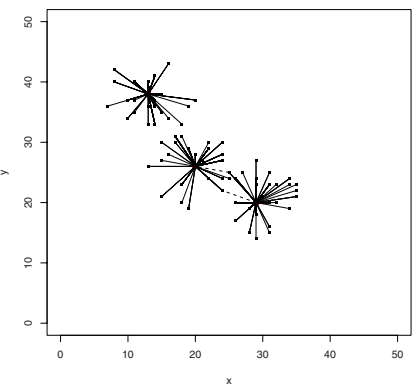

(d)

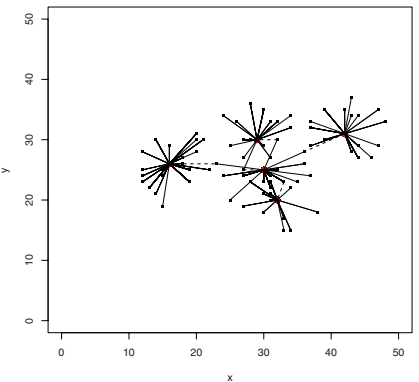

Fig. 2. Four representations of immune shape space at the end of exposure to five antigen genotypes $\left(\mathcal{R}_{1, . .4}\right)$. The emergence of the $\alpha$-indexes is indicated by the dashed edges appearing first when the system has been exposed to three antigens (b), with $\mathcal{R}_{4}$. At exposure to five antigens (d), with $\mathcal{R}_{4}$, the number of $\alpha$-vertices is five, and all five of the subgraphs are linked to form one.

\section{Results}

Fig. 2 shows the state of shape space at the end of primary response. Progressive exposure to varied antigens (values drawn from $\mathcal{R}$ ) is shown from (a) to (d). In (d), the model has been exposed to 5 different and unrelated antigenic challenges, in much the same way that a maturing immune system would be at the end of 3-4 years of development. The only parameter varied during simulation execution is $\mathcal{R}$. In (a) and (b), the central (or median) vertex is connected by a set of edges to leaves which appear in response to the prior appearance of the median vertices. Biologically, the infected antigen presentation cell, once recognised, triggers a process (known as clonal expansion) which eventually results in the immune system applying clearance pressure against the infected cell (and all cells presenting the same viral genetic material). In (b), when the system has been exposed to two infections, the subgraphs remain unconnected, indicating that no clearance pressure is applied cross-reactively, and there are no $\alpha$-vertices. At the point of third antigen challenge, (c), there emerges two $\alpha$-vertices (acting on the lower two subgraphs). The edges connecting the $\alpha$-vertices are shown as 


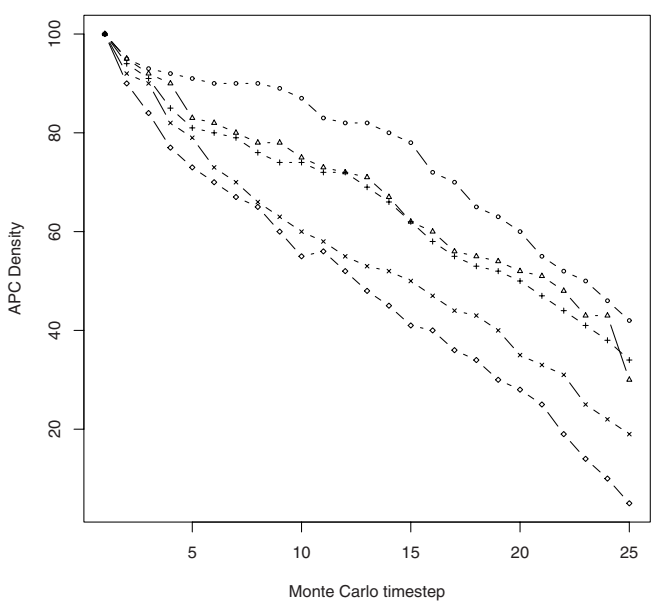

Fig. 3. Clearance rates of $a p c^{+}$for $\mathcal{R}_{4}$, under five different regimes of $\alpha$-vertex disruption: 1 to 5 (respectively $\diamond \times+\triangle$ and $\circ$ ), sampled 25 times during model execution and representing some 6 days of real-world time. Selective knock-out (or disruption due to pathogen mutation) of an $\alpha$-vertex reduces the efficacy of infected cell detection and clearance. When all five vertices have been disrupted (shown by the circle), only $\approx 60 \%$ of infected cell clearance takes place. In all cases, the $\beta$-vertex response remained healthy. In viral pathogens known to mutate slowly (influenza) or quickly (HIV), immune memory (our $\alpha$-vertices) can often be less effective (as the viral target drifts in shape space). The figure shown here offers some insight into consequences of memory cell disruption.

a broken line. At this point, the third infection to appear has caused an activation pattern which results in cross-reactive pressure applied against it. This pressure (by way of the $\alpha$-vertex) is significant, because it does not emerge over the normal time period of clonal expansion (usually some $3-5$ days). Rather, the pressure is applied instantaneously as the $\alpha$-vertices exist a priori and are therefore primed in advance. When four infections have been experienced by the system (Fig. 2(c)), three of the subgraphs have merged into one, by way of some $4 \alpha$-vertices. When the fifth unrelated infection is encountered by the system, a complex network of subgraphs, connected by some $5 \alpha$-vertices, has coalesced into one graph. Once the subgraphs merge, the dynamical immune process of up- and down regulation may be explained as follows:

(i) The appearance of $m_{1}$ stimulates the development of both $\alpha$ - and $\beta$ - vertices in $R$.

(ii) In turn, $\alpha$-vertex acts to reduce (or down-regulate) $m_{2}$

(iii) The down-regulation of $m_{2}$ causes a down regulation in $\beta$-vertices of $Q$ (as their source of stimulation declines) 
And this process may be summarised thus:

$$
\uparrow m_{1} \Rightarrow \uparrow\{\alpha, \beta \in R\} \Rightarrow \downarrow m_{2} \Rightarrow \downarrow\{\beta \in Q\}
$$

Once $\alpha_{\text {crit }}=5$ has been reached, the removal of any further infections which may arise is achieved by way of the rapid appearance of $\alpha$-vertices, and rapid increase in edge density of the connected, weighted graph $G$ in shape space. In Fig. 3 , the relative importance of the $\alpha$-vertices over the $\beta$-vertices is shown. This figure shows the model clearance rate of $A P C^{+}$from the lymphatic compartment during exposure to five infections. However, in each case, we have explored the effects of disrupting $\alpha$-vertices. Disruption will occur whenever an $A P C^{+}$ drifts from its original shape space coordinates $(\Delta x, \Delta y)$. Drift is likely to arise whenever an $\mathrm{APC}^{+}$mutates (for example, in the case of HIV). Disruption to one or two $\alpha$-vertices does not seriously degrade clearance, but results in an average reduction of efficiency of $\sim 15 \%$. This is intuitive: the importance of the $\alpha$-vertex lies not in one individual but in the cumulative effect of all. Further disruption results in progressively worse clearance ability. Disruption of all five $\alpha$-vertices reduces infected cell clearance by around $35 \%$. We would not expect a total immune failure even under full disruption, as there still remains a healthy and effective $\beta$-vertex response. Each simulation is repeated 30 times, and the results are averaged. The results shown here were obtained from five separate model simulation runs (for $\mathcal{R}_{4}$ ), at each stage, a further $\alpha$-vertex was suppressed and the edges connecting subgraphs consequentially did not emerge.

\section{Discussion and Conclusions}

In this research we have provided an outline of how the immune system shape space may be usefully extended to model the process by which infectious agents may be targeted by cells which have been primed in response to a previous and unrelated infection. Using an approach based on graph theory, we identified two qualitatively different vertex types: $\alpha$ and $\beta$. Although both vertex types form part of the cellular effector response, we have shown that an effective immune response depends largely on successful $\alpha$-vertex activation for efficacious response, and only to a lesser extent, on $\beta$-vertices. We have seen how disruption to $\alpha$-vertex activation results in a suppressed response characteristic of chronic infection. In the results presented we have proposed that $\alpha$-vertices have strong biological equivalent: cytotoxic lymphocyte memory cells. Such cells, having been primed by way of previous immune challenge, require less time to respond, and, crucially, tend to be beneficially cross-reactive. This finding supports [12] and others. Disruption to $\alpha$-vertices results in a significantly degraded pathogen clearance than disruption to $\beta$-vertices does. This supports the theory that during primary and secondary response, some cytotoxic genotypes are more important than others 12 . The network implication of $\alpha$-vertices is that each edge connection formed from a median vertex to an $\alpha$-vertex acts as a back-bone in joining disparate subgraphs. As these subgraphs connect, two related further questions arise (i) up and down regulation of competing $C T L^{+}$across subgraphs 
and (ii) the emergence of a balanced graph ${ }^{3}$. We have already shown how upand down- regulation may be explained by extending shape space as an evolving graph (or network).

\section{References}

1. Burns, J., Ruskin, H.: A Model of Immune Suppression and Repertoire Evolution. In: Sloot, P.M.A., Gorbachev, Y.E., (eds.): Lecture Notes in Computer Science, Vol. 2660. Springer-Verlag, Berlin Heidelberg New York (2003) 75-85

2. Albert, R., Barabasi, A.: Topology of Evolving Networks: Local Events and Universality. Phys. Rev. Lett. 85(24) (2000) 5234-5237

3. Buseyne, F., and Riviere, Y.: The flexibility of the TCR allows recognition of a large set of naturally occurring epitope variants by HIV-specific cytotoxic T lymphocytes Int. Immunol. (13) (1999) 941-950

4. Burns, J., Ruskin, H.: Viral Strain Diversity and Immune Response - a Computational Model. In: Hamza, M.H., (ed): Proceedings of the IASTED International Conference, Biomedical Engineering. ACTA Press (2003) 60-65

5. Perelson, A.S., Oster, G.F.: Theoretical Studies of Clonal Selection: Minimal Antibody Repertoire Size and Reliability of Self-Non-Self Discrimination. J. Theor. Biol. 81(4) (1979) 645-670

6. de Boer, R.J., Segel, L.A., Perelson, A.S.: Pattern formation in one- and twodimensional shape-space models of the immune system. J. Theor. Biol. 155(3) (1992) 295-333

7. Smith, D., Forrest, S.: Deriving shape space parameters from immunological data. J. Theor. Biol. 189 (1997) 141-150.

8. Janeway, C.A., Travers, P., Walport, M., Capra, J.D.: Immunobiology. The Immune System in Health and Disease. 4th edn. Churchill-Livingston (1999)

9. Wick, D.: The Disappearing CD4+T Cells in HIV Infection: a Case of Overstimulation?. J. Theor. Biol. 197 (1998) 507-516

10. Diestel, R.: Graph Theory. 2nd edn. Spinger (1997)

11. Wuchty, S., Stadler, P.: Centers of complex networks. J. Theor. Biol. 223 (2003) 45-53

12. Brehm, M.A., Pinto, A.K., Daniels, K.A., Schneck, J.P., Welsh, R.M., Selin, L.K.: $\mathrm{T}$ cell immunodominance and maintenance of memory regulated by unexpectedly cross-reactive pathogens Nat. Immunol. 3(7) (2002) 627-634

${ }^{3}$ A graph $G$ is balanced if the maximum ratio of edges to vertices, taken over all subgraphs of $G$, occurs at $G$ itself 